Original Research Paper

\title{
Painting Psychotherapy Image Measurement and Reconstruction Algorithm based on Improved Hadamard Matrix
}

\author{
${ }^{1 *}$ Min Wan, ${ }^{1}$ Qiang Yang and ${ }^{2}$ Huajun Wang \\ ${ }^{1}$ College of Computer and Information Engineering, Yibin University, China \\ ${ }^{2}$ College of Geophysics, Chengdu University of Technology, China
}

\author{
Article history \\ Received: $12-08-2020$ \\ Revised: 03-11-2020 \\ Accepted: 05-11-2020 \\ Corresponding Author: \\ Min Wan \\ College of Computer and \\ Information Engineering, \\ YiBin University, China \\ Email: wanmin@yibinu.edu.cn
}

\begin{abstract}
Painting psychotherapy based on Internet technology has become a method of mental health testing and treatment. This method will produce a large number of digital images of painting psychotherapy. How to measure and reconstruct the image is the basis of automatic analysis and processing. This study aimed to propose a painting psychotherapy image measurement and reconstruction algorithm based on the improved Hadamard matrix. Moreover, the construction of a measurement matrix was analyzed using the compressed sensing theory and the Hadamard matrix construction algorithm was improved and optimized. Also, four different measurement matrices were compared and analyzed. The findings revealed that the improved Hadamard measurement matrix achieved good experimental results in terms of signal reconstruction accuracy and Peak Signal-to-Noise Ratio (PSNR) value. In the experimental analysis of painting psychotherapy image reconstruction, the reconstruction algorithm based on the improved Hadamard measurement matrix had a shorter reconstruction time and the algorithm achieved satisfactory results in terms of the number of iterations and the PSNR value of the reconstructed image. For painting psychotherapy images, the algorithm provided a theoretical basis for later digital processing, large-sample training and analysis and automatic machine recognition and judgment.
\end{abstract}

Keywords: Compressed Sensing, Image Measurement and Reconstruction, Improved Hadamard Measurement Matrix, Painting Psychotherapy

\section{Introduction}

Psychologists combine painting and psychology to form the research direction of painting therapy and psychotherapy. Researchers have described and expressed different psychological states with different image features and achieved good results in reducing emotions, alleviating mental symptoms and performing psychological consultation or evaluation (Schwartz, 2018).

However, in the implementation process, manual paintings could not fully reflect a participant's real psychological state due to limitations in terms of the participant's painting level and environmental conditions. This shortcoming led to parameter deviation in the analysis of painting psychotherapy image reconstruction, thereby changing the effect of painting psychotherapy.
In recent years, psychologists have started using representative image models of houses, trees, people and other psychological characteristics with the development of mobile Internet technology, software technology and intelligent terminals. This advancement has allowed participants to assemble and draw through intelligent terminals or computers (Bouchoucha et al., 2019). Further, the digital image expression of painting psychotherapy can be realized and big data samples and network interaction platforms are possible for painting psychotherapy. A research foundation for machine learning and intelligent processing of painting psychotherapy is thus established.

In the process of painting psychotherapy based on digital image analysis and processing, this study aimed to analyze and set forth a feature measurement and reconstruction algorithm to better extract the features and 
semantic expression of painting psychotherapy images and facilitate image storage, transmission and feature analysis. The improved measurement matrix had fewer independent random variables. The constructed measurement matrix had better sparsity, which was suitable for feature analysis and intelligent processing of painting psychotherapy images.

This study analyzes the Hadamard measurement matrix, construction algorithm and optimization algorithm and then analyzes the image measurement and reconstruction algorithm of painting psychology. Finally, the experimental results are compared and analyzed.

\section{Hadamard Measurement Matrix}

A Hadamard matrix is defined as follows (Jiang et al., 2013): For an $N \times N$ order of the matrix $H_{n}$, the elements of the matrix $H_{n}$ are all +1 or -1 . If the matrix $H_{n}$ satisfies the conditions given in Equation (1), the matrix $H_{n}$ is a Hadamard matrix:

$$
H_{n} H_{n}^{T}=H_{n}^{T} H_{n}=n I_{n}
$$

Most of the methods for constructing a Hadamard matrix are based on the number theory (Huckle and Kravvaritis, 2017). The construction methods include the Turyn construction method, Paley algorithm first construction method, second Paley Williamson construction method and the Sylvester algorithm method. The graph theory hypercube concept is also used to construct a Hadamard matrix (Lan and Min, 2013).

A hypercube is constructed by $2^{n}$ points in the $n$ dimensional space. Points $2^{n}$ are recorded as $\left\{x_{0}, x_{1}\right.$, $\left.x_{2}, \ldots x_{2^{n}-1}\right\}$; if the distance between the points $x_{i}, x_{j}$ meets $\sqrt{\left(x_{i}-x_{j}\right)^{2}}=1$, then the edge of the cube is determined and the length of the edge is marked as 1 . According to the relationship between the vertices and edges of the hypercube (Rychkov, 2019), the matrix of the undirected graph is obtained as follows:

$$
\left[\begin{array}{ccccc}
0 & 0 & \ldots & 0 & 0 \\
0 & 0 & \ldots & 0 & 1 \\
0 & 0 & \ldots & 1 & 0 \\
\ldots & \ldots & \ldots & \ldots & \ldots \\
1 & 1 & \ldots & 1 & 1
\end{array}\right]
$$

The shortest distance between two points is calculated according to the representation matrix of the hypercube undirected graph; the distance between two points is marked as $d_{i j}$; if $d_{i j}=1$ or $d_{i j}=2$, the $H_{i j}$ of the corresponding matrix is 1 ; if $d_{i j}$ is the other value, then $H_{i j}$ is labeled as -1 .

\section{Improved Hadamard Measurement Matrix and Rapid Construction Method}

A previous study (Peng et al., 2018) found that any subset of the column vectors in a perfect measurement matrix was orthogonal; the larger the column of the quantum set, the better the measurement matrix. According to this principle, a random block Hadamard matrix (Scrambled Block Hadamard Ensemble, SBHE) was designed in that study. The sampling value was obtained for each sample to achieve the difference of +1 and -1 . That is:

$$
y_{i}=y_{i}(+1)-y_{i}(-1)
$$

In the actual sampling of the medical image, only the +1 portion was obtained. Considering that the total energy of the medical image was equal to the sum of +1 and -1 and the total energy in the sampling time was constant, Equation (3) could be transformed as follows:

$y_{i}=2 y_{i}(+1)-y_{\varepsilon}$

A random block Hadamard matrix was used in medical image sampling. First, the measurement matrix $\Phi$ was used to sample the signal in which the measurement matrix $\Phi$ was an element of all " 1 " matrices and the sampled values were represented by $y_{\varepsilon}$. Then, the set of random block Hadamard matrices was used for sampling the signal and obtaining an intermediate sampling value $y_{i}(+1)$. The sampling value $y_{\varepsilon}$ and intermediate sampling value $y_{i}(+1)$ were substituted into Equation (4) to obtain the final sampling value $y_{i}$.

The present study proposed improvements to the Hadamard measurement matrix and the rapid construction method using the Galois field characteristic function and random block Hadamard matrix.

Let $l$ be an odd prime number. A mod operation was performed for an integer $x(x \bmod l)$. In the remainder of $l-1$, if $m^{2} \equiv x(\bmod ) l$, then $x$ was two times the remainder. For an odd prime number $l$, Equation (5) was used to solve the Galois field problem (Li et al., 2006):

$G F\left(l^{r}\right)=\left\{0, x^{0}, x, x^{2}, \ldots, x^{l^{r}-2}\right\}$

In Equation (5), $r$ is a positive integer and $x$ is the root element Gal of the Galois field $G F\left(l^{r}\right)$. In the Galois field $G F\left(l^{r}\right)$, Equation (6) was used to solve the characteristic function $k(x)$ :

$k(x)= \begin{cases}0, & \text { if } x=0 \\ 1, & \text { if xisthetwo residue } \\ 1, & \text { if xis not the two residue }\end{cases}$ 
In this study, the Hadamard measurement matrix was constructed to determine the position of "1" and "-1" using the value of $k(x)$. The values of " 1 " and "-1" were kept the same in the rows and columns of the improved Hadamard measurement matrix to ensure that the measurement matrix of the structure met the Restricted Isometry Property (RIP) criterion.

In the rapid construction algorithm of the improved Hadamard measurement matrix, the first-row and the first-column elements were set to "1." Then, a basis matrix was constructed, according to Equation (6) in the characteristic function $k(x)$, to determine the value of the fundamental matrix that first appeared in the first line of "1" and the "-1" position. The values of the other elements were the same as those of the upper left corner in the base matrix, except for the first row and the first column.

The description and implementation steps of the improved Hadamard measurement matrix and rapid construction algorithm were as follows:

Step1: According to the length of the input signal $n$ and sampling rate of $R$, the order of the matrix $M$ was determined, where $M$ was an integer close to $(n R) \times n$ and $(M-1)$ was required to meet the "odd prime" condition.

Step 2: The elements of the first row and first column were set to "1" and the $(M-1)$ matrix was represented by $\Phi$. The Hadamard measurement matrix was represented as $\left[\begin{array}{cc}1 & I \\ I^{T} & \Phi\end{array}\right]$, where $I$ is the $(M-1)$-dimensional row vector.

Step 3: According to Equation (6), the positions of "1" and " -1 " in the first row and first column of the matrix $\Phi$ were determined.

Step 4: In the matrix $\Phi$, the values of the other elements were the same as those of the upper left corner, except the first row and first column and the structure of the improved Hadamard measurement matrix was constructed by the cyclic shift transformation.

According to the improved Hadamard measurement matrix algorithm, the fourth-order Hadamard measurement matrix was constructed as follows:

$$
\left[\begin{array}{cccc}
1 & 1 & 1 & 1 \\
1 & -1 & 1 & -1 \\
1 & -1 & -1 & 1 \\
1 & 1 & -1 & -1
\end{array}\right]
$$

According to the improved Hadamard measurement matrix algorithm, the eighth-order Hadamard measurement matrix was constructed as follows:

$$
\left[\begin{array}{cccccccc}
1 & 1 & 1 & 1 & 1 & 1 & 1 & 1 \\
1 & 1 & 1 & -1 & 1 & -1 & -1 & -1 \\
1 & -1 & 1 & 1 & -1 & 1 & -1 & -1 \\
1 & -1 & -1 & 1 & 1 & -1 & 1 & -1 \\
1 & -1 & -1 & -1 & 1 & 1 & -1 & 1 \\
1 & 1 & -1 & -1 & -1 & 1 & 1 & -1 \\
1 & -1 & 1 & -1 & -1 & -1 & 1 & 1 \\
1 & 1 & -1 & 1 & -1 & -1 & -1 & 1
\end{array}\right]
$$

According to the improved Hadamard measurement matrix algorithm, the 12th-order Hadamard measurement matrix was constructed as follows:

$$
\left[\begin{array}{cccccccccccc}
1 & 1 & 1 & 1 & 1 & 1 & 1 & 1 & 1 & 1 & 1 & 1 \\
1 & -1 & 1 & -1 & 1 & 1 & 1 & -1 & -1 & -1 & 1 & -1 \\
1 & -1 & -1 & 1 & -1 & 1 & 1 & 1 & -1 & -1 & -1 & 1 \\
1 & 1 & -1 & -1 & 1 & -1 & 1 & 1 & 1 & -1 & -1 & -1 \\
1 & -1 & 1 & -1 & -1 & 1 & -1 & 1 & 1 & 1 & -1 & -1 \\
1 & -1 & -1 & 1 & -1 & -1 & 1 & -1 & 1 & 1 & 1 & -1 \\
1 & -1 & -1 & -1 & 1 & -1 & -1 & 1 & -1 & 1 & 1 & 1 \\
1 & 1 & -1 & -1 & -1 & 1 & -1 & -1 & 1 & -1 & 1 & 1 \\
1 & 1 & 1 & -1 & -1 & -1 & 1 & -1 & -1 & 1 & -1 & 1 \\
1 & 1 & 1 & 1 & -1 & -1 & -1 & 1 & -1 & -1 & 1 & -1 \\
1 & -1 & 1 & 1 & 1 & -1 & -1 & -1 & 1 & -1 & -1 & 1 \\
1 & 1 & -1 & 1 & 1 & 1 & -1 & -1 & -1 & 1 & -1 & -1
\end{array}\right]
$$

The improved Hadamard measurement matrix had the following advantages: It had fewer independent random variables, the improved method could be realized by a fast cyclic shift transform and the measurement matrix was sparser.

\section{Measurement Matrix Optimization}

The measurement matrix was required to satisfy the RIP criterion in the compressed sensing theory. In practice, RIP is often used instead of "irrelevant." Therefore, the correlation should be minimized in the construction of the measurement matrix. The minimization of "correlation" was the main criterion for the optimal design of the measurement matrix (Li and Qin, 2011).

Correlation reflected the maximum of the crosscorrelation between the columns of the $(M \times N)$ dimensional measurement matrix $\Phi$. The optimization of the measurement matrix based on the cross-correlation involved the selection of $N$ vectors from the $M$ dimensional space; the maximum of the cross-correlation between the $N$ vectors was the minimum. The best case for cross-correlation could be considered when the maximum of each column of the $M$-dimensional space reached the Welch lower bound (Huihui et al., 2014). 
The optimal design of the measurement matrix changed the relationship between the elements in $\Phi$. Given the sparsity of $K$, a certain number of measurements were required to restore the signal $M$ minimum. The objective of the measurement matrix was to reconstruct the original signal correctly using the measurement vector $M$ and the sparsity of the signal $K$ was the maximum.

A good correlation was found between each column of the Hadamard matrix and good signal reconstruction ability existed. The row-orthogonal, row-mode and column-mode were kept the same to retain the advantages of the Hadamard matrix. In this study, the improved Hadamard matrix was optimized and the optimization algorithm was as follows:

Step 1: The improved Hadamard matrix was represented as $H$, the number of iterations was set to $I$, the initial value of $I$ was set to 0 and the number of iterations of the maximum $I$ was set to $I_{\max }$. The correlation coefficient between each column vector of the matrix $H$ was represented by $\varphi_{c i}$ and the correlation coefficient between each vector matrix was denoted by $\varphi_{r i}$. Each column-vectormode was represented by norm $_{c i}$ and each rowvector-mode was represented bynorm ${ }_{r i}$.

Step 2: $\varphi_{c i}$ and $\varphi_{r i}$ were calculated $^{[11]}$ and the maximum value $\varphi_{\text {cimax }}$ of the absolute value of the correlation coefficient between the column vectors and the maximum value $\varphi_{\text {rimax }}$ of the absolute value of the correlation coefficient between the row vectors were determined.

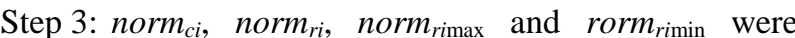
calculated.

Step 4: Each vector matrix in $H$ was normalized for the orthogonal matrix, followed by normalization of the unit vector of the column matrix in $H$.

Step 5: The number of iterations $I$ was increased by 1 ( $I$ $=I+1)$ to determine whether $I$ reached the maximum value of $I_{\max }$; if $I>I_{\max }$, then the optimized measurement matrix $H^{\prime}$ was output; otherwise, step 2 through step 5 were repeated.

\section{Image Measurement and Reconstruction Algorithm of Painting Psychotherapy}

In this study, the algorithm of painting psychotherapy image measurement and reconstruction based on the improved Hadamard matrix was designed using the principle of the Orthogonal Matching Pursuit (OMP) algorithm (Nguyen and Park, 2018). The idea of the medical image reconstruction algorithm was as follows:

$\theta \in R^{N}$ was a $K$-sparse vector, that is, the $\theta$ of the $N$ elements had only $K$-nonzero elements. The signal $X \in R^{N}$ could be expressed as $X=\Psi \theta, Y \in R^{N}, n<N$, where $Y$ was obtained by improving the Hadamard measurement matrix, that is:

$Y=H X$

The improved Hadamard measurement matrix satisfied the RIP criterion and the medical image reconstruction could resolve the following optimization problems $\left(\min \|\theta\|_{1}\right)$, which was equivalent to finding the optimal sparse solution $Y=H \Psi \theta$.

The signal $X$ was sparse and compressible and hence the problem of solving the undetermined equations $Y=$ $A^{C S} X$ was converted into an $l_{1}$ optimization problem:

$\min \left\|\Psi^{T} X\right\|_{1} \quad$ s.t. $A^{C S} X=H \Psi^{T} X=Y$

$x_{\Gamma^{n}}$ could be estimated by solving unconstrained optimization problems:

$\min \left\|y-H_{\Gamma^{n}} x_{\Gamma^{n}}\right\|_{2}^{2}$

Equation (9) was a new way to optimize the problem. A new way of estimating $x_{\Gamma^{n}}$ was:

$x_{\Gamma^{n}}^{n}=x_{\Gamma^{n}}^{n-1}+a^{n} d_{\Gamma^{n}}^{n}$

In Equation (10), $d_{\Gamma^{n}}^{n}$ represents the direction of the iterative update and $a^{n}$ represents the step size. $a^{n}$ could be calculated as:

$a^{n}=\frac{<r^{n}, H_{\Gamma^{n}} d_{\Gamma^{n}}^{n}}{\left\|H_{\Gamma^{n}} d_{\Gamma^{n}}^{n}\right\|_{2}^{2}}$

The flow chart of image measurement and reconstruction algorithm of painting psychology is shown in Fig. 1.

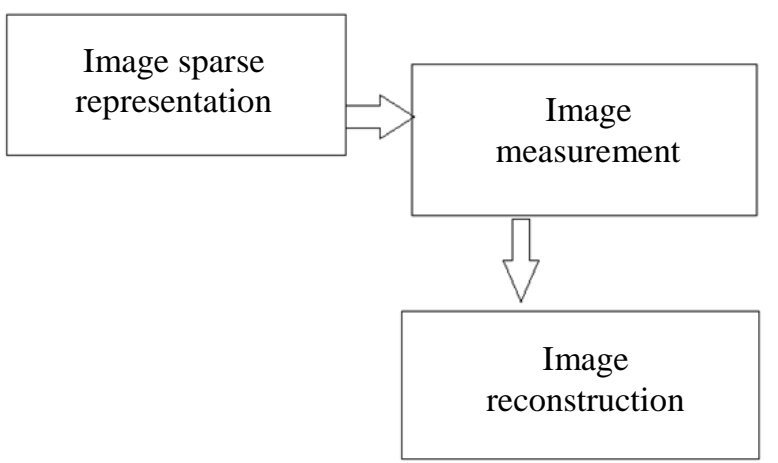

Fig. 1: The flow chart of image measurement and reconstruction algorithm 
According to the OMP algorithm (Bahaghighat and Motamedi, 2017), the image reconstruction algorithm based on the improved Hadamard measurement matrix was described and implemented as follows:

Step 1: Suppose $H$ is an improved Hadamard measurement matrix, $Y$ is the observation vector, $K$ is the sparsity, $r$ and $r^{0}=Y$ are the initialization residuals, $x^{0}=0, \Gamma^{0}=\varnothing$ are the initialization parameters and $n$ is the number of iterations, which was set to 0 .

Step 2: $X$ was the re-established sparse solution and $I$ was the index set, with $I=E \cdot g^{n}=\left\langle n^{r-1}, H\right\rangle$ was calculated.

Step 3: $i^{n}=\arg \max \left|g_{i}^{n}\right| \quad$ was $\quad$ calculated and $\Gamma^{n}, \Gamma^{n}=\Gamma^{n-1} \cup i^{n}$ was updated.

Step 4: $a^{n}$ was updated according to Equation (12):

$$
a^{n}=\frac{<r^{n}, H_{\Gamma^{n}} d_{\Gamma^{n}}^{n}}{\left\|H_{\Gamma^{n}} d_{\Gamma^{n}}^{n}\right\|_{2}^{2}}
$$

Step 5: $x_{\Gamma^{n}}, x_{\Gamma^{n}}^{n}=x_{\Gamma^{n}}^{n-1}+a^{n} d_{\Gamma^{n}}^{n}$ was estimated.

Step 6: $r^{n}, r^{n}=r^{n-1}-a^{n} H_{\Gamma^{n}} d_{\Gamma^{n}}^{n}$ was updated.

Step 7: The iteration condition was determined; if $\left|r^{n}-r^{n-1}\right| \leq 10^{-6}$, then the iteration was terminated and the $r^{n}$ and $x_{\Gamma^{n}}^{n}$ were output. If unsatisfied with the iterative termination conditions, step 2 through step 7 was performed again.

\section{Experimental Analysis}

In this study, the Peak Signal-to-Noise Ratio (PSNR) was used to evaluate the effect of the reconstructed image.
The PSNR was calculated as follows (Bahaghighat and Motamedi, 2017):

$$
P S N R=10 \lg \frac{\left(I^{\max }{ }_{i, j}\right)^{2}}{\frac{1}{M N} \sum_{(i, j)}\left(I_{i, j}-Z_{i, j}\right)^{2}}
$$

In Equation (13), $0 \leq i \leq M-1,0 \leq j \leq N-1, I^{\max }{ }_{i, j}$ is $255, I_{i, j}$ is the pixel value of the source image coordinate position $(i, j)$ and $Z_{i}, j$ is the pixel value of the reconstructed image coordinate position $(i, j)$.

\section{Experimental Analysis of Measurement Matrix Construction Algorithm}

In the simulation experiment, two $(256 \times 256)$-pixel painting psychotherapy images were selected as the test images. Image G1 was the experimental image of houses, trees and people taken by the camera, while image G2 was the construction image of houses, trees and people drawn by the participants using computers. The two original images are shown in Fig. 2.

For the test image, this study adopted the Gaussian random matrix, Hadamard matrix, SBHE measurement matrix and the improved Hadamard measurement matrix to perform the sampling and reconstruction experiments.

Figure 3 is the angiographic image G1 according to the $16 \times 16$ block, with a sampling rate of $0.20,0.25,0.35$ and 0.40 and reconstruction of the painting psychotherapy image used to obtain the PSNR value.

In Fig. 2, the reconstruction algorithm obtained a large value for reconstruction of the painting psychotherapy image G1 using the same sampling rate. The reconstruction experiment achieved good results.

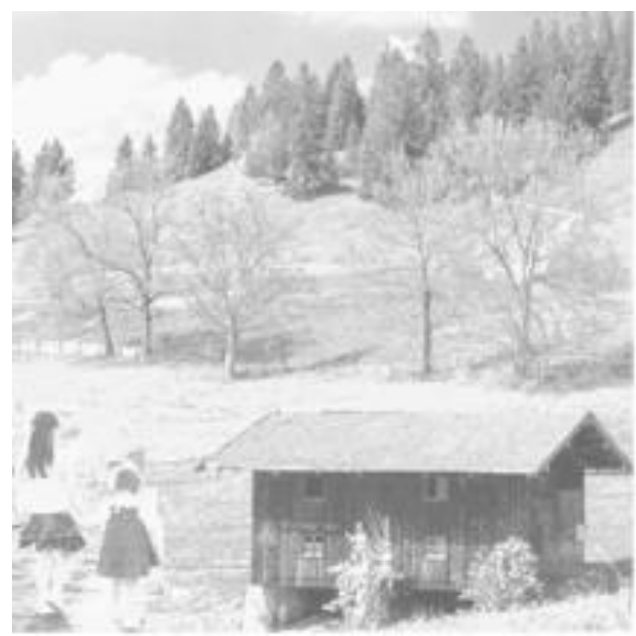

(a)

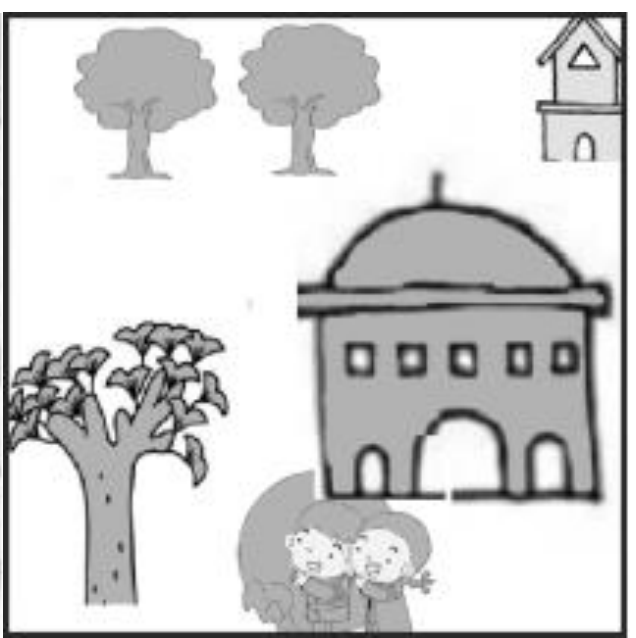

(b)

Fig. 2: Test images; (a) Image G1 taken with a camera. (b) Image G2 drawn by a participant 


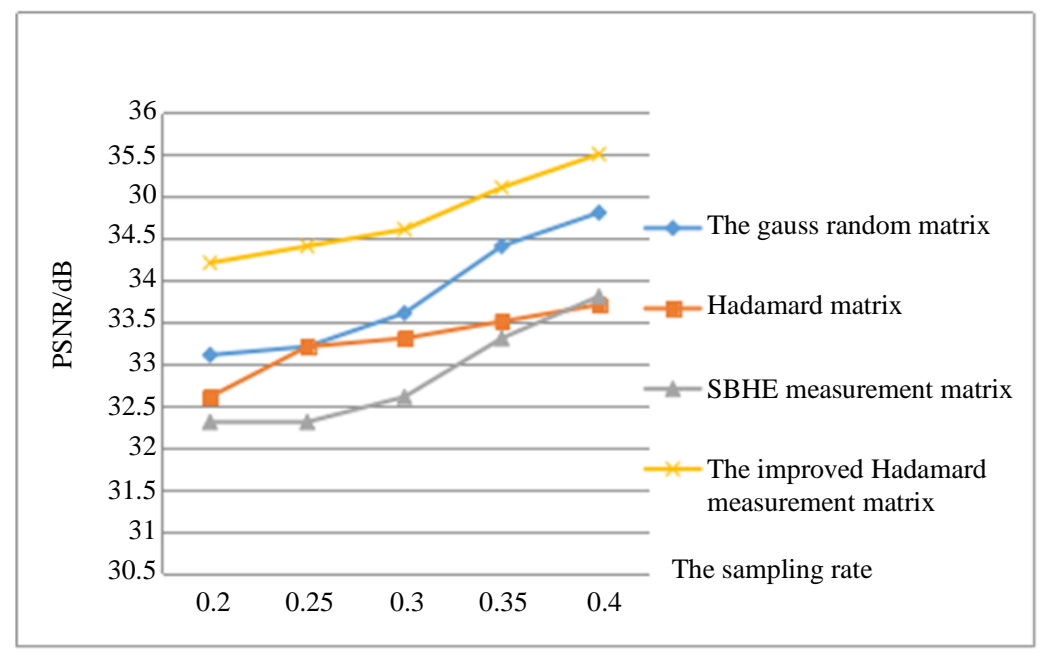

Fig. 3: PSNR value for the reconstruction of painting psychotherapy image G1

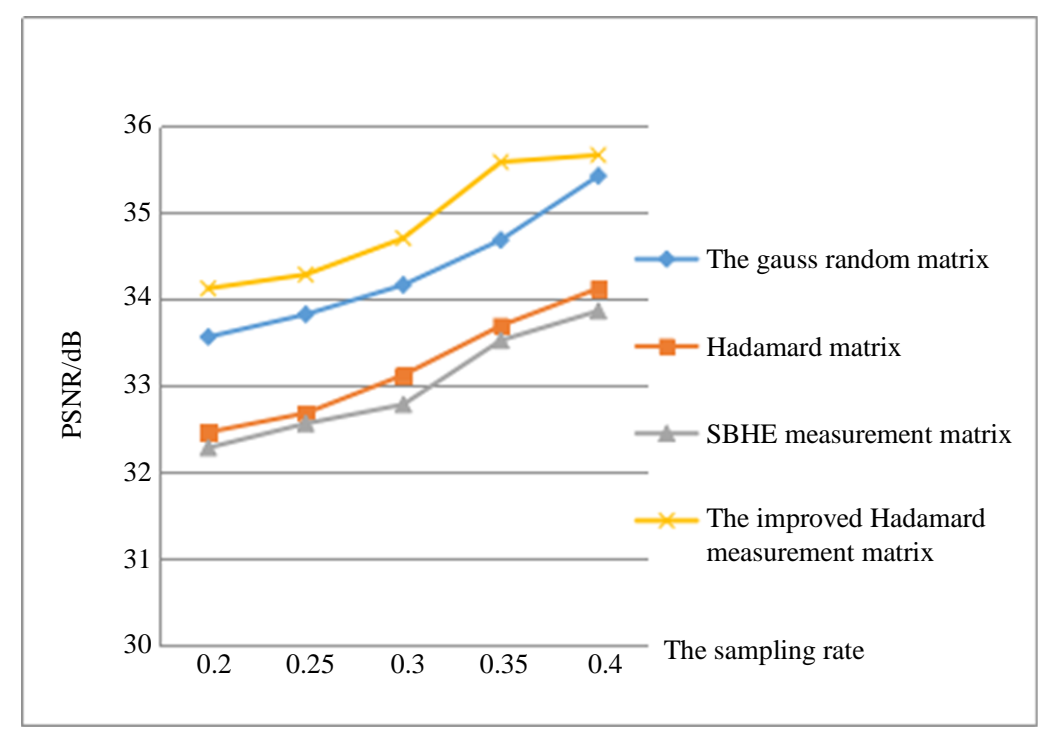

Fig. 4: PSNR value for the reconstruction of image G2 drawn by a participant

In Fig. 4, the reconstruction algorithm obtained a large PSNR value for the reconstruction of image G2 drawn by a participant using the same sampling rate. The reconstruction experiment achieved good results.

According to the PSNR value of the reconstructed image, the value of the improved Hadamard measurement matrix for image G2 drawn by a participant was higher at different sampling rates and the reconstructed image had a better PSNR value.

Four different matrices were tested using the OMP reconstruction algorithm further to analyze the relationship between reconstruction probability and sparsity. The Gauss sparse signal was used as a test and the measurement matrix was evaluated using a $256 \times 256$ measurement matrix. This matrix was used to test the signal 100 times with different sparsity signals. The test data are shown in Fig. 5.
According to the data in Fig. 5, the improved Hadamard measurement matrix had a higher probability of reconstruction under the condition of the same degree of sparsity and the optimized Hadamard measurement matrix had a better reconstruction effect in the OMP reconstruction algorithm.

\section{Experimental Analysis of Painting Psychotherapy Image Reconstruction Algorithm}

The images G1 and G2 were chosen for experimental analysis in painting psychotherapy image reconstruction algorithm experiments. Image G1 was the experimental image of houses, trees and people taken with the camera, while image $\mathrm{G} 2$ was the construction image of houses, trees and people drawn by the participants using computers. The PSNR was used as the criterion for judging the 
reconstructed images. In painting psychotherapy image reconstruction algorithm experiments, the sparse representation of the angiographic image G1 was based on the wavelet transform and the improved Hadamard matrix was used as the measurement matrix. The reconstruction algorithms were as follows: The OMP algorithm based on the Gradient Projection for Sparse Reconstruction (GPSR),
Basis Pursuit (BP) algorithm, Bregman algorithm and the improved algorithm. The PSNR value of the reconstructed image G1, at sampling rates of $0.20,0.30,0.40,0.50,0.60$ and 0.70 , is shown in Table 1 . The reconstruction algorithm was based on the OMP algorithm, GPSR algorithm, BP algorithm, Bregman iterative algorithm and the improved algorithm proposed in this study.

Table 1: PSNR value of the reconstructed image G1

\begin{tabular}{lllllll}
\hline Sampling rate & 0.2 & 0.3 & 0.4 & 0.5 & 0.6 & \\
\hline OMP & 30.5 & 31.2 & 32.1 & 33.5 & 35.2 & 36.8 \\
GPSR & 31.4 & 31.5 & 33.1 & 34.1 & 34.5 & 36.3 \\
BP & 30.6 & 32.1 & 33.2 & 33.4 & 34.6 & 35.7 \\
Bregman iterative algorithm & 30.5 & 32.3 & 33.1 & 34.5 & 35.1 & 36.5 \\
Improved algorithm & 30.8 & 32.6 & 33.3 & 34.6 & 35.8 & 37.1 \\
\hline
\end{tabular}

Table 2: Number of iterations (G2) with different sparsity (n/every time)

\begin{tabular}{lcccr}
\hline Number of iterations & Sparsity 120 & Sparsity 160 & Sparsity 200 & Sparsity 240 \\
\hline OMP & 103 & 164 & 198 & 235 \\
GPSR & 19 & 22 & 25 & 32 \\
Bregman iterative algorithm & 26 & 27 & 46 & 52 \\
Improved algorithm & 18 & 20 & 23 & 26 \\
\hline
\end{tabular}

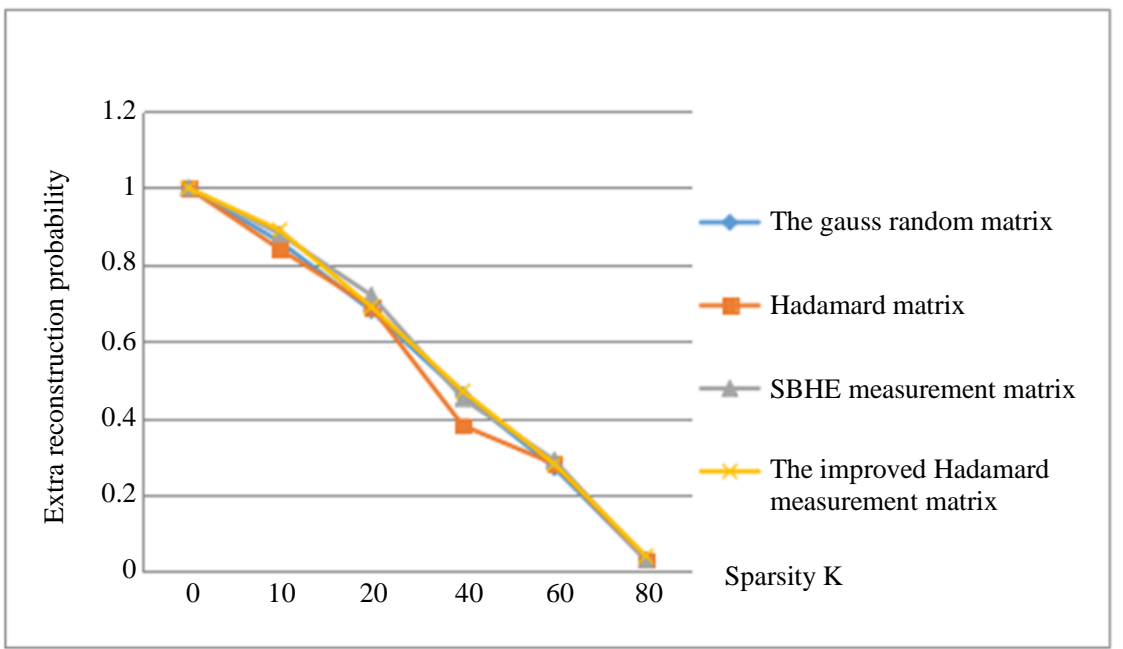

Fig. 5: Relationship between the probability of reconstruction and sparsity

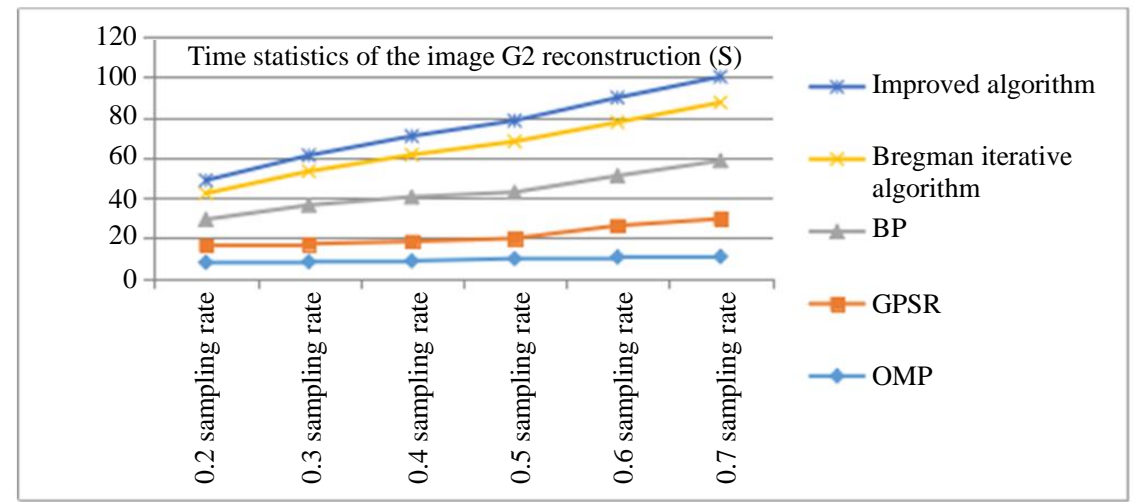

Fig. 6: Reconstruction time of the reconstruction algorithms 


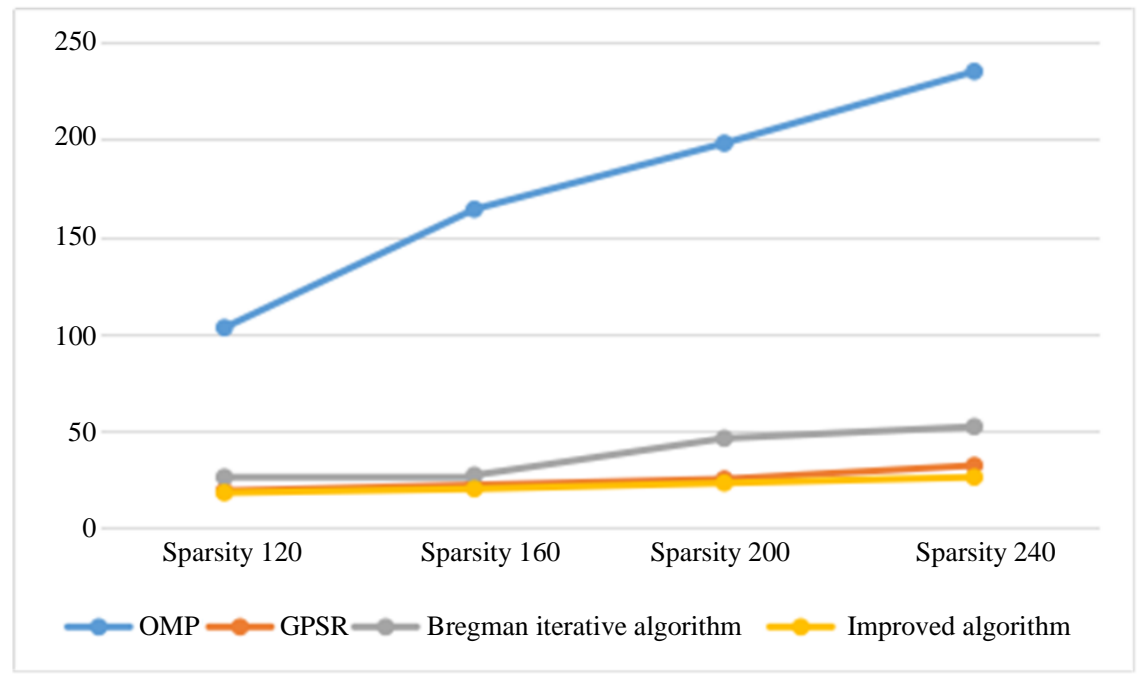

Fig. 7: Iterative analysis of the reconstruction algorithm

The time statistics of the reconstruction of image G2, at sampling rates of $0.20,0.30,0.40,0.50,0.60$ and 0.70 , are shown in Fig. 6. The reconstruction algorithm was based on the OMP algorithm, GPSR algorithm, BP algorithm, Bregman iterative algorithm and the improved algorithm proposed in this study, showing that the improved algorithm had the lowest reconstruction time.

Table 2 shows the results of experiments using different reconstruction algorithms on the image G2 under different sparsity conditions $(120,160,200$ and 240). The reconstruction was set at the same mean square error and the number of iterations is shown in Table 2.

Figure 7 shows the number of iterations of each algorithm. Each algorithm had a different sparsity but the same reconstruction mean square error conditions.

The experimental results showed that the painting psychotherapy image reconstruction algorithm proposed in this study achieved satisfactory results in terms of the PSNR value of the reconstructed image, number of iterations and execution time of the reconstruction algorithm.

\section{Conclusion}

In this study, both the construction of the measurement matrix in the compressed sensing theory and the construction algorithm of the Hadamard matrix were analyzed. The improvement and optimization of the Hadamard measurement matrix, as well as the painting psychotherapy image measurement and reconstruction algorithm based on the improved Hadamard matrix, were proposed.

A comparison of four different measurement matrices showed that the proposed algorithm achieved good results.
In the painting image reconstruction experiment, the painting psychotherapy image reconstruction algorithm based on improved Hadamard measurement matrix achieved satisfactory results in terms of the number of iterations, reconstruction time and PSNR value of the reconstructed image. For painting psychotherapy images, the algorithm provided a theoretical basis for later digital processing, large-sample training and analysis and automatic machine recognition and judgment.

\section{Acknowledgment}

This study was supported by the Scientific Research Fund of Sichuan Provincial Education Department (No:17ZB0493) and the Research Fund Project of Yibin University (2019YY04).

\section{Author's Contributions}

Wan Min: Designed and performed the experiments, analyzed the data and prepared the paper.

Yang Qiang: Participated to collect the materials related to the experiment.

Wang Huajun: Designed the experiments and revised the manuscript.

\section{Ethics}

Authors should address any ethical issues that may arise after the publication of this manuscript.

\section{References}

Bahaghighat, M., \& Motamedi, S. A. (2017). Psnr enhancement in image streaming over cognitive radio sensor networks. Etri Journal, 39(5), 683-694. 
Bouchoucha, M., Girault-Lidvan, N., Hejnar, M., Mary, F., Airinei, G., \& Benamouzig, R. (2019). Clinical and psychological characteristics of patients with globus. Clinics and research in hepatology and gastroenterology, 43(5), 614-622.

Huckle, T. K., \& Kravvaritis, C. D. (2017). Properties of submatrices of Sylvester Hadamard matrices. Linear and Multilinear Algebra, 65(8), 1629-1642.

Huihui, L., Yan, Z., Ning, Y., Xiwen, Y., \& Linhong, Q. (2014). Improved compressed sensing reconstruction algorithm and its application in image fusion. Journal of Nanjing University of Science and Technology, 38(02), 259-263.

Jiang, L. B., Huang, T., Shen, H. N., \& Liu, Z. Z. (2013). Orthogonal multi matching pursuit algorithm based on local randomized Hadamard matrix. Syst. Eng. Electron, 35(5), 914-919.

Lan, W., \& Min, W. (2013). A hadrmard matrix construction method based on hypercube graph. Wuyi University Journal. 32(2), 51-54.

Li, H. H., Guo, L., \& Liu, H. (2006). Research on image fusion based on the second generation curvelet transform. ActaOpticaSinica, 26(5), 657-662.
Li, X., \& Qin, S. Y. (2011). Efficient fusion for infrared and visible images based on compressive sensing principle. IET Image Processing, 5(2), 141-147.

Nguyen, V. Q., \& Park, S. Y. (2018). High-performance ASIC realization of orthogonal matching pursuit algorithm. IEICE Electronics Express, 15-20180075.

Peng, J., Li, L., \& Tang, Y. Y. (2018). Maximum likelihood estimation-based joint sparse representation for the classification of hyperspectral remote sensing images. IEEE transactions on neural networks and learning systems, 30(6), 1790-1802.

Rychkov, K. L. (2019). On the Perfectness of Minimal Regular Partitions of the Edge Set of the nDimensional Cube. Journal of Applied and Industrial Mathematics, 13(4), 717-739.

Schwartz, K. M. (2018). Making unformulated experience real through painting: Painting and psychoanalytic psychotherapy practice as two ways of making sense. Journal of clinical psychology, 74(2), 239-248. 